\title{
Are Novel Cardiac Biomarkers Required in Prediction of Heart Failure Development and Outcomes?
}

\author{
Alexander E. Berezin, Professor, MD, PhD. \\ Consultant of Therapeutic Unit, Private Hospital "Vita-Center", Zaporozhye, Ukraine \\ Consultant of Therapeutic Unit, Department of Internal Medicine, Medical University, Zaporozhye, \\ Ukraine
}

\begin{abstract}
Heart failure remains a global burden for patients with established cardiovascular (CV) disease. It has been postulated that underlying mechanisms of nature evolution of HF might be identified by a measurement of some biomarker level that reflects various pathophysiological stages of cardiac dysfunction evolution. On this way, cardiac biomarkers, which affect biomechanical stress, cardiac injury, fluid overload, inflammatory reaction, may be useful tool for prediction of development, progression, and prognosis of HF. The short communication is depicted to a discussion around perspectives to use in routine HF clinical practice new biomarkers, i.e. procalcitonin, copeptin, heart-type fatty acid-binding protein; growth differentiation factor 15. It has concluded that these biomarkers are needed to be investigated in details, while there is suggestion that multiple biomarker models would be better in prediction HF evolution and outcomes than even single brand new biomarker.
\end{abstract}

Keywords: chronic heart failure; biomarkers; procalcitonin, copeptin, heart-type fatty acid-binding protein; growth differentiation factor 15; prediction.

Heart failure remains a leading cause of premature death in patients with established cardiovascular (CV) disease [1]. Prevalence of HF has been exhibiting a strong tendency to growth worldwide. Although there are several clinical guidelines regarding diagnosis, prevention and treatment of HF, prediction of HF development in various patient populations is still under scientific discussion. Biological markers have become a powerful tool for stratification ofHF patients at risk and biomarker-guided therapy [2].Updated clinical recommendations have been reported that the natriuretic peptides, galectin-3, high-sensitivity troponin and soluble ST2 protein are commonly used biomarkers, which remain a central part of routine clinical practice to stratify patients at risk of HF development, arisk of primary admission / readmission to the hospital, and CV death. Confusingly, the role of biomarkers in modification of treatment care considerably relates to aging, CV disease and metabolic co-morbidities, kidney clearance, and higher individual biological variability of biomarkers, which negatively effects on interpretation of circulating biomarkers' level[3].In this context, novel biomarkers are required to assist in the titration of medical therapy and improve prediction of widely used scores [4].

Procalcitonin, copeptin, heart-type fatty acid-binding protein (hFABP) and growth differentiation factor 15 (GDF-15) has been suggested to be novel biomarkers in HF.

Procalcitoninis known a precursor of the calcitonin, which is produced and actively secreted by the parafollicular C cells of the thyroid gland and involved in regulation of calcium homeostasis [5]. Recent clinical studies have shown that procalcitonin as an inflammatory biomarker had a pretty accurate diagnostic ability to sepsis, shock, bacterial complications of some diseases [6-8]. Additionally, this biomarker may help to manage the patients with HF when antibiotic use is needed or the critical state has been verified [4]. However, there is not strong evidence regarding procalcitotin use in biomarker-guided therapy to adjust dosage of drugs for HF individuals.

Copeptin is C-terminal peptide derived from the precursor molecule of arginine vasopressin, which plays a pivotal role in fluid retention and electrolyte homeostasis [9]. In the general population elevated level of copeptin strongly associated with increased CV mortality [10]. Additionally, based 
on results of serial measurements of copeptin level it has been suggested that the increased copept in concentration or trend to elevation of one area independent risk factor for long-term HF-related clinical outcomes and sudden death in patients with established CV disease [11-13].Being able to better predict all-cause mortality rate and HF-related risks including death and admission to the hospital copeptin might be considered as much more accurate biomarker than natriuretic peptides for optimize medical care in HF patients [14, 15]. Unfortunately, there are large body of evidence regarding that the level of copeptin might relate closely to some metabolic abnormalities including hyperglycemia that sufficiently limits the predictive power of the biomarker in serial measurements especially in patients with diabetes and obesity $[14,16]$. However, the improvement of diagnostic reliability of copeptin may achieve by means use of combined biomarker strategy, in particular it might be based on copeptin and natriuretic peptides ( $\mathrm{N}$-terminal pro-brain natriuretic peptide, midregional pro-atrial natriuretic peptide) [17, 18]. Finally, circulating level of copeptinare now recognized a promising biomarker with better discriminative value for both all-cause mortality and HF-related outcomes general population and individuals with established CV disease.

The main biological role of heart type of FABP (hFABP) is to facilitate the long-chain fatty acids reuptake, attenuate calcium transport in cardiomyocytes and regulate inflammatory response in reply to some lipid signals [19]. hFABP is predominantly expressed in cardiomyocytes and is powerful biomarker of myocardial injury. Recent studies have shown that the hFABP has better predicted CV outcomes to other biomarkers of cardiac damage, i.e. myoglobin and high-sensitive troponins $[3,14$, 20], whereas elevated intestinal FABP would identify patients with advanced HF who had severe fluid retention and intestinal congestion [21]. Overall, the H-FABP may better provide prognostic information on survival and more precise reflecta risk of major $\mathrm{CV}$ events during hospitalization period and short-time after discharge than natriuretic peptides, cardiac troponins and galectin-3. However, the role of several types of FABP in HF is not fully clear. Large clinical studies are required to more accurately explain the predictive value of these biomarkers.

Growth differentiation factor (GDF)-15 belongs to the super family of transforming growth factor- $\beta$ [22]. GDF-15 is widely expressed on the surfaces of various cells. In HF GDF-15 is secreted by injured cardiomyocytesin response to ischemia, reperfusion, inflammatory cytokine stimulation and exposure to biomechanical stress [14]. Elevated level of circulating GDF-15 was found in HF individuals irrespectively etiology of cardiac dysfunction [23]. There is strong evidence regarding being tight interrelationship between circulating level of GDF-15 and HF signs and symptoms, reduced left ventricular ejection fraction [24]. Although serial biomarker evaluation has not showed superiority of incremental predictive ability in GDF-15 versus natriuretic peptides in acute HF [25], in chronic HF multiple marker strategy based on GDF-15, galectin-3 and natriuretic peptides might exhibit several advantages before conventional approach in ability to predict all-cause mortality, CV mortality and HF-related outcomes in outpatients with HF [26, 27]. Finally, there are several controversies regarding importance of predictive value for survival and incremental prognostication in diagnosis of HF. There is need in larger clinical studies with higher statistical power and head-to-head comparison of biomarkers to clear their role in diagnosis and guided therapy of HF.

Conclusions: Although recent clinical trials have been exhibited much more information regarding biomarker use in prognostication of HF, there is considerable limitation in head-to-head comparison of several biomarkers and biomarker-based strategy to treat of HF. All these are a cause of some speculations around advantages and shortcomings of biomarker-based management of HF including new biological indicators, such as procalcitonin, copeptin, hFABP and GDF-15. Novel biomarkers are needed to be investigated in details, while there is suggestion that multiple biomarker models would be better in prediction HF evolution and outcomes than even single brand new biomarker.

\section{REFERENCES}

[1] Ponikowski P, Voors AA, Anker SD, Bueno H, Cleland JG, Coats AJ, et al. Authors/Task Force Members. 2016 ESC guidelines for the diagnosis and treatment of acute and chronic heart failure: the Task Force for the diagnosis and treatment of acute and chronic heart failure of the European Society of Cardiology (ESC) developed with the special contribution of the Heart Failure Association (HFA) of the ESC. Eur Heart J. 2016;37:2129-2200.

[2] Wettersten N, Maisel AS. Biomarkers for Heart Failure: An Update for Practitioners of Internal Medicine. Am J Med. 2016; 129(6):560-7. 
[3] Berezin AE. Prognostication in different heart failure phenotypes: the role of circulating biomarkers. Journal of Circulating Biomarkers. 2016, 5:01 doi: 10.5772/62797.

[4] Pouleur AC. Which biomarkers do clinicians need for diagnosis and management of heart failure with reduced ejection fraction? ClinChim Acta.2015;443: 9-16.

[5] Ryu JA, Yang JH, Lee D, Park CM, Suh GY, Jeon K, et al. Clinical Usefulness of Procalcitonin and C-Reactive Protein as Outcome Predictors in Critically Ill Patients with Severe Sepsis and Septic Shock. PLoS One. 2015. 10 (9):e0138150

[6] Reiner MM, Khoury WE, Canales MB, Chmielewski RA, Patel K, Razzante MC, et al. Procalcitonin as a Biomarker for Predicting Amputation Level in Lower Extremity Infections. J Foot Ankle Surg. 2017 doi: 10.1053/j.jfas.2017.01.014. [Epub ahead of print]

[7] Hayashida K, Kondo Y, Hara Y, Aihara M, Yamakawa K. Head-to-head comparison of procalcitonin and presepsin for the diagnosis of sepsis in critically ill adult patients: a protocol for a systematic review and meta-analysis. BMJ Open. 2017;7(3):e014305.

[8] Simon L, Gauvin F, Amre DK, Saint-Louis P, Lacroix J. Serum procalcitonin and C-reactive protein levels as markers of bacterial infection: a systematic review and meta-analysis. Clin Infect Dis 2004;39:206-17

[9] Morgenthaler NG. Assay for the measurement of copeptin, a stable peptide derived from the precursor of vasopressin. Clin Chem. 2006; 52, 112-119

[10] Remde H, Dietz A, Emeny R, Riester A, Peters A, de Las Heras Gala T et al. The cardiovascular markers copeptin and high-sensitive C-reactive protein decrease following specific therapy for primary aldosteronism. J Hypertens. 2016; 34, 2066-73.

[11] Moayedi Y, Ross HJ. Advances in heart failure: a review of biomarkers, emerging pharmacological therapies, durable mechanical support and telemonitoring. ClinSci (Lond). 2017;131(7):553-566.

[12] Krane V, Genser B, Kleber ME, Drechsler C, März W, Delgado G, et al. Copeptin Associates with Cause-Specific Mortality in Patients with Impaired Renal Function: Results from the LURIC and the 4D Study. Clin Chem. 2017 doi: 10.1373/clinchem.2016.266254. [Epub ahead of print]

[13] Yan JJ, Lu Y, Kuai ZP, Yong YH. Predictive value of plasma copeptin level for the risk and mortality of heart failure: a meta-analysis. J Cell Mol Med. 2017. doi: 10.1111/jcmm.13102. [Epub ahead of print]

[14] Berezin AE. Biological markers of cardiovascular diseases. Part 4. Diagnostic and prognostic value of biological markers at risk stratification among patients with heart failure. LAMBERT Academic Publishing GmbH, Moskow, 2015. - 329 p.

[15] Savic-Radojevic A, Pljesa-Ercegovac M, Matic M, Simic D, Radovanovic S, Simic T. Novel Biomarkers of Heart Failure. AdvClin Chem. 2017; 79: 93-152.

[16] Smaradottir MI, Ritsinger V, Gyberg V, Norhammar A, Näsman P, Mellbin LG. Copeptin in patients with acute myocardial infarction and newly detected glucose abnormalities - A marker of increased stress susceptibility? A report from the Glucose in Acute Myocardial Infarction cohort. DiabVasc Dis Res. 2017;14(2): 69-76.

[17] Sahin I, Gungor B, Ozkaynak B, Uzun F, Küçük SH, Avci II, et al. Higher copeptin levels are associated with worse outcome in patients with hypertrophic cardiomyopathy. ClinCardiol. 2017; 40(1): 32-37.

[18] Herrero-Puente P, Prieto-García B, García-García M, Jacob J, Martín-Sánchez FJ, Pascual-Figal $\mathrm{D}$, et al. Predictive capacity of a multimarker strategy to determine short-term mortality in patients attending a hospital emergency Department for acute heart failure. BIO-EAHFE study. ClinChimActa. 2017;466: 22-30.

[19] Chmurzynska A. The multigene family of fatty acidbinding proteins (FABPs): function, structure, and polymorphism. J Appl Genet. 2006; 47, 39-48.

[20] Qian HY, Huang J, Yang YJ, Yang YM, Li ZZ, Zhang JM. Heart-type Fatty Acid Binding Protein in the Assessment of Acute Pulmonary Embolism. Am J Med Sci. 2016; 352(6):557-562. 
[21] Kitai T, Kim YH, Kiefer K, Morales R, Borowski AG, Grodin JL, et al. Circulating intestinal fatty acid-binding protein (I-FABP) levels in acute decompensated heart failure. ClinBiochem. 2017. doi: 10.1016/j.clinbiochem.2017.02.014. [Epub ahead of print]

[22] Kempf T, Wollert KC. Growth Differentiation Factor-15: a New Biomarker in Cardiovascular Disease. Herz. 2009; 34, 594-9.

[23] Chan MM, Santhanakrishnan R, Chong JP, Chen Z, Tai BC, Liew OW, et al. Growth differentiation factor 15 in heart failure with preserved vs. reduced ejection fraction. Eur J Heart Fail. 2016;18(1):81-8.

[24] Hage C, Michaëlsson E, Linde C, Donal E, Daubert JC, Gan LM, et al. Inflammatory Biomarkers Predict Heart Failure Severity and Prognosis in Patients With Heart Failure With Preserved Ejection Fraction: A Holistic Proteomic Approach. CircCardiovasc Genet. 2017;10(1). doi: 10.1161/CIRCGENETICS.116.001633.

[25] Demissei BG, Cotter G, Prescott MF, Felker GM, Filippatos G, Greenberg BH, et al. A multimarker multi-time point-based risk stratification strategy in acute heart failure: results from the RELAX-AHF trial. Eur J Heart Fail. 2017. doi: 10.1002/ejhf.749. [Epub ahead of print]

[26] Berezin AE, Kremzer AA, Martovitskaya YV, Samura TA, Berezina TA, Zulli A, et al. The utility of biomarker risk prediction score in patients with chronic heart failure. Int J ClinExp Med 2015;8(10): 18255-64.

[27] Berezin AE. The risk stratification in heart failure patients: The controversial role of highsensitive ST2. J IntegrCardiol, 2015; 1(6): 216-217.

Citation: Alexander E. Berezin. Are Novel Cardiac Biomarkers Required in Prediction of Heart Failure Development and Outcomes?. ARC Journal of Cardiology. 2017; 3(1):17-20.

Copyright: $\odot 2017$ Alexander E. Berezin. This is an open-access article distributed under the terms of the Creative Commons Attribution License, which permits unrestricted use, distribution, and reproduction in any medium, provided the original author and source are credited.. 